\title{
Analysis of Quantitative Trait Loci for Resistance to Southern Leaf Blight in Juvenile Maize
}

\author{
P. J. Balint-Kurti and M. L. Carson
}

First author: U.S. Department of Agriculture-Agricultural Research Service (USDA-ARS) Plant Science Research Unit, North Carolina State University, Raleigh 27695; and second author: USDA-ARS, Cereal Disease Laboratory, St. Paul, MN 55108. Accepted for publication 15 October 2005.

\section{ABSTRACT}

Balint-Kurti, P. J., and Carson, M. L. 2006. Analysis of quantitative trait loci for resistance to southern leaf blight in juvenile maize. Phytopathology 96:221-225.

A set of 192 maize recombinant inbred lines (RILs), derived from a cross between the inbred lines Mo17 and B73, were evaluated as 3-weekold seedlings in the greenhouse for resistance to southern leaf blight, caused by Cochliobolus heterostrophus race O. Six significant (LOD >3.1) quantitative trait loci (QTL) were identified for disease resistance, located on chromosomes 1, 2, 3, 6, 7, and 8. Results were compared with a previous study that had used the same RIL population and pathogen iso- late, but had examined resistance in mature rather than juvenile plants. There was a very weak but significant correlation between the overall resistance phenotypes of the RILs scored as mature and juvenile plants. Two QTL were found in similar positions on chromosomes 1 and 3 at both growth stages. Other QTL were specific to one growth stage or the other. Twenty-three of these RILs, together with the parental lines, were inoculated in the greenhouse with four $C$. heterostrophus isolates. Results indicated that the quantitative resistance observed was largely isolate nonspecific.

Additional keyword: corn.
Cochliobolus heterostrophus (Drechs.) Drechs. (anamorph = Bipolaris maydis (Nisikado) Shoemaker; synonym = Helminthosporium maydis Nisikado) is a necrotrophic plant pathogen and the causal agent of southern leaf blight (SLB). This disease is widely found in hot, humid maize-growing areas but was not considered an important pathogen until 1970 when C. heterostrophus race $\mathrm{T}$ became prevalent in the U.S. corn belt. Race $\mathrm{T}$ was highly pathogenic on Texas male-sterile cytoplasm (cms-T) and caused a major epidemic in 1970 and 1971 (37). Since that time, cms-T has been eliminated from elite germ plasm and effective polygenic resistance has been introduced. Most of this resistance is quantitative and additive or recessive in effect $(5,24,27$, 34,36 ), although one qualitative recessive gene, $r h m$, which primarily conditions resistance in pre-anthesis growth stages, has been mapped to the distal end of the short arm of chromosome six (bin 6.00) (40). The disease, predominantly caused by race $O$, is still a problem in sweet corn and seed production and in the southern Atlantic coast area of the United States on field maize. It can cause grain yield losses of $40 \%$ or more $(6,18,22,37)$.

Most U.S. maize hybrids rely primarily on some form of quantitative resistance to control a variety of diseases. Many qualitative plant resistance genes have been cloned (23) and their downstream pathways characterized $(23,32)$, but little is known about the molecular genetic basis or mechanisms of action of quantitative plant disease resistance genes. Numerous studies have been published on the mapping of disease resistance quantitative trait loci (QTL) to particular genomic regions $(3,4,13,14)$. While

Corresponding author: P. J. Balint-Kurti; E-mail address: peter_balintkurti@ncsu.edu

* The $\boldsymbol{e}$-Xtra logo stands for "electronic extra" and indicates that the online version contains supplemental material not included in the print edition. Figure 1 appears in color online.

DOI: $10.1094 /$ PHYTO-96-0221

This article is in the public domain and not copyrightable. It may be freely reprinted with customary crediting of the source. The American Phytopathological Society, 2006. knowledge of QTL is useful for, among other things, marker assisted breeding, QTL can generally be located with confidence within, at minimum, a $20 \mathrm{cM}$ window, which gives little clue as to the molecular identity of the genes involved. Scoring for any quantitative trait on an individual plant basis is difficult and is often inconsistent across environments $(4,31)$, making gene cloning techniques such as transposon tagging and map-based cloning challenging. Most cases in which a quantitative gene has been successfully identified $(15,17,19,21)$ involved some combination of relatively easily scorable phenotype, defined environmental conditions, and the construction and use of near-isogenic lines, facilitating the phenotypic scoring of the QTL in a segregating population.

Field evaluation of quantitative resistance can be considered the ultimate reference against which other evaluation techniques are measured. In the field, mature plants can be assessed under natural disease conditions and multiple infection cycles. However, a laboratory or greenhouse-based assay can be a useful additional tool as it allows for greater environmental and inoculum standardization (both in terms of isolate identity and amount) and should enable a more reproducible assessment of resistance phenotype. Multiple experiments a year can be performed in the greenhouse, whereas in the field, there is usually only one opportunity annually. In addition, a greenhouse inoculation technique could be used in conjunction with other experimental approaches such as expression profiling. However, it is not clear that greenhouse studies with maize, which use juvenile plants, will detect resistance mechanisms that function in mature plants in the field. Since field resistance is the goal of our research, it was important to answer this question.

The main objective of this study was to determine whether, at a gross level, resistance in juvenile and mature plants was related and, at a finer level, whether loci that are important for quantitative resistance in mature plants in the field might also be important for resistance in juvenile plants. If loci are identified that function in both environments, one can then proceed with the analysis of these loci in the greenhouse, in the expectation that 
any findings will be relevant to the more practically important situation of mature plants in the field.

\section{MATERIALS AND METHODS}

Plant materials. Phenotypic data were collected from a mapping population comprised of $192 \mathrm{~F}_{6: 7}$ recombinant inbred lines (RILs) that were derived from the cross of maize inbred lines B73 (relatively susceptible parent) and Mo17 (relatively resistant parent) and from the two parental lines. Genotypic information for this population had previously been generated at 234 restriction fragment length polymorphisms, isozymes, and simple sequence repeat marker loci (35).

Plant growth conditions. Experimental units consisted of two plants of a particular line grown in a single 15 -cm-diameter clay pot filled with a 50:50 mixture of Metromix (Scott's Inc., Marysville, $\mathrm{OH}$ ) and sterilized soil. Supplemental lighting was used during winter months to maintain $\mathrm{a} \approx 16 \mathrm{~h}$ day length, approximately $14 \mathrm{~h} /$ day during January and $10 \mathrm{~h} /$ day in February. The plants were maintained at $26^{\circ} \mathrm{C}$ during the day and $22^{\circ} \mathrm{C}$ during the night. The actual temperature rarely deviated more than $4^{\circ} \mathrm{C}$ from these target temperatures.

Fungal growth, inoculation, and rating techniques. $C$. heterostrophus isolate $2-16 \mathrm{Bm}$ was used for the QTL studies. Three additional isolates (Hm28, 9-31Bm, and NI1011) were used for the investigation of line-isolate interactions. Isolates were grown on potato dextrose agar plates at $25^{\circ} \mathrm{C}$ under a $12 \mathrm{~h}$ light/dark cycle. Spores were collected by submerging 1 - to 2 -week-old cultures in water and brushing the spores free with a camel-hair brush.

Plants were inoculated using a technique based on one reported in Zhu et al. (41). Water containing $0.05 \%$ agar and $0.25 \%$ Tween 20 was used to make a $5 \times 10^{3} \mathrm{ml}$ conidial suspension. The suspension was sprayed as a fine mist until runoff onto both surfaces of the youngest fully expanded leaf of 21-day-old (i.e., 21 days from planting) seedlings at the five-leaf stage. Inoculated plants were placed in a humidity tent for 12 to $16 \mathrm{~h}$ overnight and then replaced on the greenhouse bench. Leaves were rated for percent necrotic leaf area 6 days after inoculation.

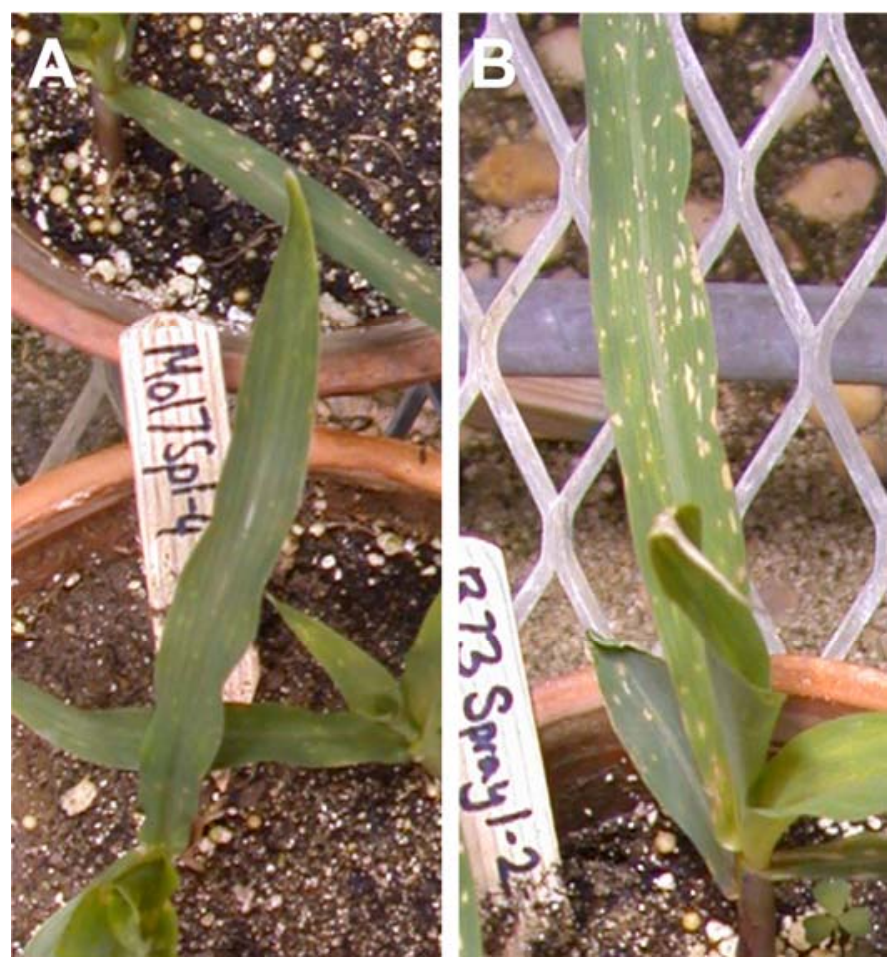

Fig. 1. A, Mo17 and B, B73 maize lines inoculated at 3 weeks after planting using spray inoculation, 6 days postinoculation.
Experimental design. Four replicates of the mapping population plus the parents were screened. Replicates 1 and 2 were screened in January 2004, replicates 3 and 4 in February 2004. A randomized complete block design was used in all cases.

The inoculations for the isolate-line interaction experiment took place in May 2004. A set of 25 lines were inoculated with four different isolates of $C$. heterostrophus race $\mathrm{O}(\mathrm{Hm} 28$, 9$31 \mathrm{Bm}, 2-16 \mathrm{Bm}$, and NI1011), all isolated from samples collected in North Carolina. The lines consisted of the parent lines (B73 and Mo17) and 23 RILs, selected from among lines for which significant amounts of seed were available, to include lines displaying high, moderate, and low resistance based on the previous greenhouse assays. The $C$. heterostrophus isolates were chosen from among those examined in a previous study (8). Ten isolates from this study had been maintained frozen as glycerol stocks at $-80^{\circ} \mathrm{C}$. Initial experiments were performed to identify suitably aggressive isolates (lesions produced within 4 days after inoculation). From among these relatively aggressive isolates, four were chosen to represent the two different mating types. The number of 25 maize lines was chosen for logistical reasons. It was considered important that all the isolates were inoculated on the same day within a relatively short period of time, to minimize any possible environmental interactions. This would not have been possible if more than 25 lines had been used. Each isolate was used to inoculate all 25 lines, arranged as a randomized complete block. Two randomized complete blocks were inoculated with each isolate. In this case, lesion lengths were measured (eight measurements per line per replication), so that the results could be better compared with those reported previously for these isolates (8).

Statistical analysis. Due to poor seed germination or growth, approximately $5 \%$ of the lines were not suitable for inoculation in each replicate. To account for missing data in QTL calculations, a least square means average for each line was calculated using the PROC GLM procedure of SAS (SAS Institute, Cary, NC), based on these four replications. All correlation calculations were made using the PROC CORR procedure of SAS.

The QTL cartographer software package (38) was used to detect the QTL. Composite interval mapping (CIM) was used. Several analyses were performed using simple interval mapping and CIM under Model 6 (the standard model), varying values for the walk speed (the genome scan interval), window size, and numbers of control markers. A CIM analysis using a walk speed of $2 \mathrm{cM}$, a window size of $2 \mathrm{cM}$, and 30 control markers, with a threshold value of 14.11 (determined by permutation analysis with a significance level of 0.05 and corresponding to a log of odds [LOD] of 3.1 ), is reported here. These conditions resulted in the detection of a relatively small number of distinct QTL peaks with relatively high LOD likelihood scores. All the QTL detected under these parameters were also consistently detected using a range of different analysis parameters.

\section{RESULTS}

Reproducibility of disease symptoms after inoculation in the greenhouse. Clear differences were observed between the resistant and susceptible parents after inoculation in the greenhouse (Fig. 1). Correlations between the four replications performed were high, with the six pair-wise Pearson correlation coefficients ranging between 0.71 and 0.79 .

Comparison of field and greenhouse resistance. The phenotypic range of least square means of necrotic leaf area observed in the recombinant inbred population was 1.2 to $79.5 \%$ (Fig. 2). Some transgressive segregation was observed with Mo17 (resistant parent) averaging $6.9 \%$ necrotic leaf area and B73 (susceptible parent) averaging $61.7 \%$ necrotic leaf area.

The correlation between the least square means found in this study with the area under disease progress curve values obtained 
previously in the field for the same lines assayed with the same $C$. heterostrophus isolate (9) was calculated. The Pearson correlation coefficient $(r)$ between these two sets of data was $0.21(P=$ $0.012)$ and Spearman rank correlation coefficient was $0.22(P=$ $0.007)$, indicating a significant but very weak correlation between the resistance phenotypes of juvenile and mature plants.

QTL identification. Significant QTL (LOD score >3.1) were found in six chromosomal bins: 1.08/09, 2.09, 3.04, 6.00, 7.02, and 8.03 (Tables 1 and 2). The resistant alleles were derived from Mo17 in four of the six cases, while the resistant alleles in bins 2.09 and 7.02 were derived from B73. These QTL together accounted for over $40 \%$ of the phenotypic variation observed. Other less significant QTL (LOD scores from 2.0 to 3.1) were detected in bins $1.06,3.06,5.07$, and 10.06/07. In each of these cases, the resistant allele was derived from Mo17. No epistatic interactions were found between any of the QTL detected.

Six major QTL were also detected in the companion field study using mature plants of the same population and inoculating with the same pathogen isolate (9). Of these QTL, only two, in bins
3.04 and 1.08/09, could be considered to have been detected in both studies (Table 2). For both QTL the resistant allele was derived from Mo17 at both growth stages.

Investigation of isolate-line interactions. The resistance phenotypes of 25 lines consisting of the RIL parents (B73 and Mo17) and 23 RILs against four different race $\mathrm{O} C$. heterostrophus isolates (Table 3) were assessed. Analysis of variance, using lines and isolates as independent variables (Table 4), showed that differences between lines were highly significant. Spearman rank correlations of lines ranked by resistance to each isolate (Table 5) ranged from 0.92 to 0.72 for the six pair-wise interactions, i.e., the ranking of lines with each isolate was quite similar, and Pearson correlation coefficients for each line-isolate combination ranged from 0.88 to 0.64 (Table 5). Although the isolates chosen showed consistent differences in the size of the lesions they formed (Table 3), they did not vary enough in aggressiveness in this limited study for significant differences between them to be observed $(P>F=0.154)$. Isolate-line interactions were nonsignificant at the $95 \%$ level $(P>F=0.054)$ (Table 4$)$.

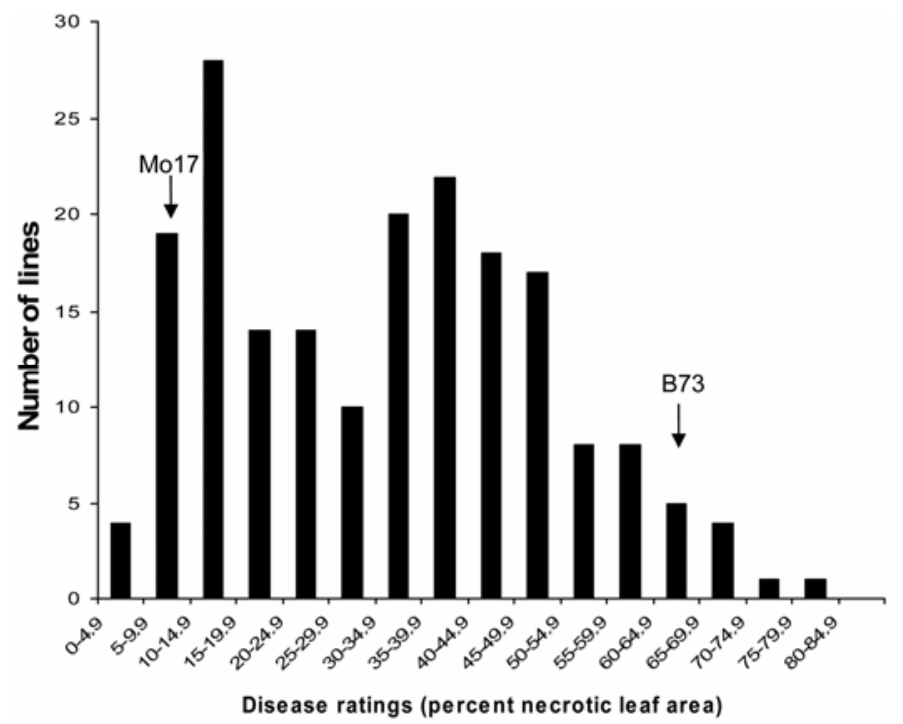

Fig. 2. The distribution of average scores for percent necrotic leaf area in the B73/Mo17 maize recombinant inbred line population. The position of the average scores of the parental types, B73 and Mo17, are indicated.
TABLE 2. The major quantitative trait loci (QTL) for resistance in maize to southern leaf blight (SLB) and associated parameters found in this study using juvenile plants (LOD score > 3.1) compared with the major QTL detected for resistance to SLB in the same population scored as mature plants in the field by Carson et al. (9) ${ }^{\mathrm{a}}$

\begin{tabular}{|c|c|c|}
\hline \multirow[b]{2}{*}{ Chromosome bin } & \multicolumn{2}{|c|}{ Associated parameters } \\
\hline & Juvenile plants & Mature plants \\
\hline 1.02 & & $R^{2}=3.2, \mathrm{a}=-31.4$ \\
\hline $1.08 / 1.09$ & $R^{2}=5.1, \mathrm{a}=4.2$ & \\
\hline $1.09 / 1.10$ & $\ldots$ & $R^{2}=24.9, \mathrm{a}=-81.3$ \\
\hline 2.04 & $\ldots$ & $R^{2}=19.8, \mathrm{a}=-71.5$ \\
\hline 2.09 & $R^{2}=3.5, \mathrm{a}=-3.3$ & $\ldots$ \\
\hline $3.03 / 3.04$ & $\ldots$ & $R^{2}=11.2, \mathrm{a}=-49.1$ \\
\hline 3.04 & $R^{2}=12.3, \mathrm{a}=6.5$ & ... \\
\hline 4.09 & $\ldots$ & $R^{2}=4.3, \mathrm{a}=-28.2$ \\
\hline 6.00 & $R^{2}=7.2, \mathrm{a}=5.1$ & $\ldots$ \\
\hline 7.02 & $R^{2}=3.2, \mathrm{a}=-3.3$ & $\ldots$ \\
\hline 8.03 & $R^{2}=10.2, \mathrm{a}=5.9$ & $\ldots$ \\
\hline 10.04 & $\ldots$ & $R^{2}=7.7, \mathrm{a}=-44.0$ \\
\hline
\end{tabular}

${ }^{a}$ Both studies used the same pathogen isolate. The additive effect is denoted by ' $a$ '. Additive values cannot be compared between studies as they were measured on different scales. This study used necrotic leaf area while Carson et al. (9) used area under disease progress curve.

TABLE 1. Location and parameters associated with major quantitative trait loci (QTL) for resistance to southern leaf blight of maize, caused by Cochliobolus heterostrophus race $\mathrm{O}$, in a B73 $\times$ Mo17 recombinant inbred (RI) line population comprising 192 lines

\begin{tabular}{|c|c|c|c|c|c|c|}
\hline Chromosome & $\mathrm{Bin}^{\mathrm{a}}$ & QTL peak flanking markers ${ }^{\mathrm{b}}$ & $\begin{array}{l}\text { One LOD interval } \\
\text { flanking markers }\end{array}$ & LOD score $^{\mathrm{d}}$ & Additive effect ${ }^{\mathrm{e}}$ & $R^{2 \mathrm{f}}$ \\
\hline 1 & 1.06 & csu3-umc133c & csu3-bnl5.59 & 2.2 & 2.8 & 1.9 \\
\hline 1 & $1.08 / 1.09$ & csu164a-phi055 & phi002-phi055 & 4.9 & 4.2 & 5.1 \\
\hline 3 & 3.04 & asg48-phi036 & asg $48-u m c 10 a$ & 12.6 & 6.5 & 12.3 \\
\hline 3 & 3.06 & bn15.37-umc60 & bn15.37-bnl6.16 & 2.5 & 2.8 & 2.3 \\
\hline 5 & 5.07 & umc108-phi048 & phi101-phi085 & 2.6 & 3.0 & 2.2 \\
\hline 6 & 6.00 & bnlg161-Rxo & bnlg161-umc163b & 7.4 & 5.1 & 7.3 \\
\hline 10 & $10.06 / 07$ & phi035-bnl7.49 & umc44a-bnl7.49 & 2.4 & 3.1 & 2.8 \\
\hline
\end{tabular}

${ }^{a}$ Chromosome bin location of QTL peak on 1 of the 10 chromosomes of the maize genome. Bins divide the genetic map into 100 approximately equal segments. The segments are designated with the chromosome number followed by a two-digit decimal (e.g., 1.00, 1.01, 1.02 and so on). The marker order determined for the population used in this experiment largely follows the marker order shown in the standard maize genetic map (the IBM map). However, there are some discrepancies.

${ }^{\mathrm{b}}$ The markers that flank the locus with the maximum log of odds (LOD) likelihood score for each QTL as determined by composite interval mapping. The left marker is the marker nearest the distal end of the short arm of the chromosome.

c The molecular markers flanking the area within one LOD value of the maximum LOD likelihood value.

$\mathrm{d}$ The maximum LOD score associated with each QTL.

${ }^{\mathrm{e}}$ The additive effect in terms of percent necrotic leaf area associated with each QTL.

${ }^{\mathrm{f}} R^{2}$ estimates the proportion of phenotypic variance (\%) explained by the detected QTL. 


\section{DISCUSSION}

The analysis of quantitative disease resistance genes presents particular challenges, as the phenotype involves interaction with another organism and is, as such, inherently more variable than those based purely on the physiology of the organism under study. In the field, other sources of variation are introduced such as a naturally occurring, mixed population of inoculum, varying in aggressiveness (8), and unpredictable environmental conditions. If detailed characterization, mapping, and cloning of quantitative disease resistance genes is to be successful, it is important to minimize the environmental variation under which data is collected. One option is to use juvenile plants in a greenhouse environment, where the isolate used for inoculation, the inoculum level, and the light and temperature levels can all be controlled.

The main objective of this work was to determine whether one can justifiably extrapolate results obtained in the greenhouse with seedlings to phenotypes expected in the field with mature plants. This is a particular concern with foliar diseases because juvenile and mature maize leaves differ for a number of characteristics such as epicuticular wax, leaf hairs, and cell structure (20). The low correlation $(r=0.21)$ between the lines scored as juvenile plants in this study and as mature plants in Carson et al. (9) indicates that the gross level of SLB resistance in juvenile plants is not highly related to the resistance level observed in the same line in the field.

At a more detailed level, of the six major QTL (LOD >3.1) detected in this study, two (in bins 1.08/9 and 3.04) coincided with or were very near two of the six significant QTL detected previously using this same population and pathogen isolate in the field study (Table 2) (9). The low but significant correlation between the lines scored as juvenile and mature plants could therefore be ascribed to these common QTL segregating with eight maturityspecific QTL. An important caveat is that even though two QTL regions do coincide in the two studies, we cannot say at this stage whether resistance is conferred by the same mechanisms or genes at the two growth stages, or whether two or more linked genes are involved, one or more at each growth stage.

TABLE 3. Cochliobolus heterostrophus isolates used for the isolate-maize line interaction studies

\begin{tabular}{|c|c|c|c|c|}
\hline \multirow[b]{2}{*}{ Isolate } & \multirow[b]{2}{*}{ Mating type } & \multicolumn{3}{|c|}{ Lesion length $(\mathrm{mm})^{\mathrm{a}}$} \\
\hline & & Greenhouse $^{b}$ & Field $^{\mathrm{c}}$ & Greenhouse (this study) $^{\mathrm{d}}$ \\
\hline $\mathrm{Hm} 28$ & 2 & 5.8 & 4.0 & 5.4 \\
\hline 9-31Bm & 1 & 5.7 & 5.9 & 3.7 \\
\hline $2-16 \mathrm{Bm}$ & 2 & 6.5 & 4.8 & 7.1 \\
\hline NI1011 & 1 & 6.5 & 5.8 & 6.2 \\
\hline
\end{tabular}

${ }^{a}$ Lesion lengths are averages of all measurements taken from greenhouse and field studies. Except for last column, all details taken from Carson (8).

${ }^{b}$ Average of measurements on the susceptible maize host Pioneer brand hybrid 3184. Details taken from Carson (8).

c Average of measurements on the maize hosts Pioneer brand hybrids 3184 and 3162. Details taken from Carson (8).

d Average of measurements on 25 maize lines: B73, Mo17, and 23 recombinant inbred lines of varying resistance. Details taken from this study.

TABLE 4. Analysis of variance of lesion length from inoculations of four Cochliobolus heterostrophus isolates on 25 different maize lines ${ }^{\mathrm{a}}$

\begin{tabular}{lrccc}
\hline Source & df & Mean square & $F$ value & $P>F$ \\
\hline Isolate & 3 & 87.0 & 3.07 & 0.154 \\
Rep(isolate) & 4 & 28.4 & 8.52 & $<0.0001$ \\
Line & 24 & 62.5 & 18.42 & $<0.0001$ \\
Isolate-line & 71 & 4.9 & 1.44 & 0.054
\end{tabular}

${ }^{\mathrm{a}}$ Isolate and line were treated as independent variables. $\mathrm{df}=$ degrees of freedom.
A significant QTL was found in bin 7.02 in this study. A QTL was also detected in the same genomic location in one of the 2 years (1995) evaluated by Carson et al. (9). However, in the present study, the juvenile plant resistance allele was derived from $\mathrm{B} 73$, whereas the mature plant resistance allele was derived from Mo17. None of the weaker QTL (LOD between 2 and 3.1) in bins 1.06, 3.06, 5.07, and 10.06/7 detected in this study co-localize with any QTL detected in the field by Carson et al. (9).

A field study of maize QTL for resistance to northern corn leaf blight (39) identified QTL at different growth stages, including in juvenile plants at the four- to six-leaf stage. As in this study, several growth stage-specific QTL were identified while other QTL were effective at a range of growth stages.

The recessive SLB resistance gene $r h m$ has been mapped to bin 6.00 (40). The effect of rhm is strongest during seedling stages though it does provide some level of resistance to mature plants post-anthesis $(36,40)$. It has been proposed that a second locus, rhm2, is located at a linked site about $10 \mathrm{cM}$ from rhml, also in bin 6.00 (11). We found a major SLB QTL in bin 6.00, while no corresponding QTL was identified in the mature plant study (9). The coincidence of position and the fact that this resistance is detected in juvenile plants but not in mature plants suggests similarities to rhm. Although Mo17 is not thought to carry rhm, it may be that it has an alternate allele of the same gene which provides more resistance than the B73 allele.

McMullen and Simcox (28) previously identified two bins, 3.04 and 6.01, as regions where many resistance loci are tightly linked. SLB QTL were identified in both these regions in this study (albeit in an adjacent bin in the case of 6.01). Bin 3.04 is of particular interest as it was identified as an SLB resistance QTL in both juvenile (this study) and mature plants (9). In addition, a previous study using tropical germplasm also identified a QTL for SLB in the vicinity of bin 3.04 (26). Furthermore, in our unpublished, ongoing studies, we have identified strong SLB resistance QTL in bin 3.04 in three populations derived from the crosses ADENT $\times$ B73, NC250 $\times$ B73, and B104 × NC300 (M. M. Goodman, M. P. Jines, D. M. Bubeck, and P. J. Balint-Kurti, unpublished data).

The question of isolate specificity of disease resistance QTL has been examined in several recent studies. In some of these, no evidence was found of isolate-specific interactions $(10,12,29,33)$, while others report finding significant isolate-line interactions or isolate-specific resistance QTL $(1,2,7,16,30)$. A previous report (25) documented the interaction between $10 \mathrm{C}$. heterostrophus isolates and 10 maize inbred lines. Although isolate-line interactions were found within individual replications, no significant isolate-line interactions were found when the data were analyzed over the six replications performed. We have also shown here that the resistance we observed is generally isolate nonspecific with respect to the four isolates examined.

TABLE 5. Correlations between southern leaf blight disease scores (percent necrotic leaf area) obtained on 25 different maize lines using four different Cochliobolus heterostrophus race $\mathrm{O}$ isolates ${ }^{\mathrm{a}}$

\begin{tabular}{lllll}
\hline & $2-16 \mathrm{Bm}$ & $9-31$ & $\mathrm{Hm} 28$ & NI1011 \\
\hline $2-16 \mathrm{Bm}$ & 1 & $\ldots$ & $\ldots$ & $\ldots$ \\
$\mathrm{I} 9-31$ & 0.81 & $\ldots$ & $\ldots$ & $\ldots$ \\
& $\underline{0.76}$ & 1 & $\ldots$ & $\ldots$ \\
$\mathrm{H} 28$ & 0.88 & $0.64^{*}$ & $\ldots$ & $\ldots$ \\
& $\underline{0.92}$ & $\underline{0.72}$ & 1 & $\ldots$ \\
NI1011 & 0.86 & 0.77 & 0.83 & $\ldots$ \\
\hline
\end{tabular}

${ }^{a}$ Pearson correlation coefficient is in normal font, and the Spearman rank correlation coefficient is underlined below. * Indicates significant at $P=$ 0.0007 . All other correlations are significant at $P<0.0001$. 


\section{ACKNOWLEDGMENTS}

This work was supported by the USDA-ARS. We thank J. Holland for his generous gift of the B73 $\times$ Mo17 RIL population and for his valuable help with data analysis; V. Brewster and H. Wilson for technical assistance; and L. Senior, R. Nelson, C. Cowger, and D. Marshall for useful discussion and critical evaluation of the manuscript.

\section{LITERATURE CITED}

1. Arru, L., Francia, E., and Pecchioni, N. 2003. Isolate-specific QTLs of resistance to leaf stripe (Pyrenophora graminea) in the 'Steptoe' $\times$ 'Morex' spring barley cross. Theor. Appl. Genet.106:668-675.

2. Avila, C. M., Satovic, Z., Sillero, J. C., Rubiales, D., Moreno, M. T., and Torres, A. M. 2004. Isolate and organ-specific QTLs for ascochyta blight resistance in faba bean (Vicia faba L). Theor. Appl. Genet. 108:10711078.

3. Brown, A. F., Juvik, J. A., and Pataky, J. K. 2001. Quantitative trait loci in sweet corn associated with partial resistance to Stewart's wilt, northern corn leaf blight, and common rust. Phytopathology 91:293-300.

4. Bubeck, D. M., Goodman, M. M., Beavis, W. D., and Grant, D. 1993. Quantitative trait loci controlling resistance to gray leaf spot in maize. Crop Sci. 33:838-847.

5. Burnette, D. C., and White, D. G. 1985. Inheritance of resistance to Bipolaris maydis race $\mathrm{O}$ in crosses derived from nine resistant inbred lines of maize, Zea mays. Phytopathology 75:1195-1200.

6. Byrnes, K. J., Pataky, J. K., and White, D. G. 1989. Relationships between yield of three maize hybrids and severity of southern leaf blight caused by race $\mathrm{O}$ of Bipolaris maydis. Plant Dis. 73:834-840.

7. Calenge, F., Faure, A., Goerre, M., Gebhardt, C., Van de Weg, W. E., Parisi, L., and Durel, C. E. 2004. Quantitative trait loci (QTL) analysis reveals both broad-spectrum and isolate-specific QTL for scab resistance in an apple progeny challenged with eight isolates of Venturia inaequalis. Phytopathology 94:370-379.

8. Carson, M. L. 1998. Aggressiveness and perennation of isolates of Cochliobolus heterostrophus from North Carolina. Plant Dis. 82:10431047.

9. Carson, M. L., Stuber, C. W., and Senior, M. L. 2004. Identification and mapping of quantitative trait loci conditioning resistance to southern leaf blight of maize caused by Cochliobolus heterostrophus race O. Phytopathology 94:862-867.

10. Castro, A. J., Chen, X., Hayes, P. M., Knapp, S. J., Line, R. F., Toojinda, T., and Vivar, H. 2002. Coincident QTL which determine seedling and adult plant resistance to stripe rust in barley. Crop Sci. 42:1701-1708.

11. Chang, R. Y., and Peterson, P. A. 1995. Genetic control of resistance to Bipolaris maydis: One gene or two genes? J. Hered. 86:94-97.

12. Chartrain, L., Brading, P. A., Widdowson, J. P., and Brown, J. K. M. 2004. Partial resistance to Septoria tritici blotch (Mycosphaerella graminicola) in wheat cultivars Arina and Riband. Phytopathology 94:497-504.

13. Clements, M. J., Dudley, J. W., and White, D. G. 2000. Quantitative trait loci associated with resistance to gray leaf spot of corn. Phytopathology 90:1018-1025.

14. Dingerdissen, A. L., Geiger, H. H., Lee, M., Schechert, A., and Welz, H. G. 1996. Interval mapping of genes for quantitative resistance of maize to Setosphaeria turcica, cause of northern leaf blight, in a tropical environment. Mol. Breed. 2:143-156.

15. Doebley, J., Stec, A., and Hubbard, L. 1997. The evolution of apical dominance in maize. Nature 386:485-488.

16. Durel, C. E., Parisi, L., Laurens, F.,Van de Weg, W. E., Liebhard, R., and Jourjon, M. F. 2003. Genetic dissection of partial resistance to race 6 of Venturia inaequalis in apple. Genome 46:224-234.

17. El-Assal, S. E.-D., Alonso-Blanco, C., Peeters, A. J. M., Raz, V., and Koornneef, M. 2001. A QTL for flowering time in Arabidopsis reveals a novel allele of $C R Y 2$. Nat. Gen. 29:435-440.

18. Fisher, D. E., Hooker, A. L., Lim, S. M., and Smith, D. R. 1976. Leaf infection and yield loss caused by four Helminthosporium leaf diseases of corn. Phytopathology 66:942-944.

19. Frary, A., Nesbitt, T. C., Frary, A., Grandillo, S., van der Knaap, E., Cong, B., Liu, J., Meller, J., Elber, R., Alpert, K. B., and Tanksley, S. D. 2000. fw2.2: A quantitative trait locus key to the evolution of tomato fruit size. Science 289:85-88.

20. Freeling, M. 1992. A conceptual framework for maize leaf development. Dev. Biol. 153:44-58.

21. Fridman, E., Carrari, F., Liu, Y. S., Fernie, A. R., and Zamir, D. 2004. Zooming In on a quantitative trait for tomato yield using interspecific introgressions. Science 305:1786-1789.

22. Gregory, L. V., Ayers, J. E., and Nelson, R. R. 1979. The influence of cultivar and location on yield loss in corn Zea mays due to southern corn leaf blight Helminthosporium maydis. Plant Dis. Rep. 63:891-895.

23. Hammond-Kosack, K. E., and Parker, J. E. 2003. Deciphering plantpathogen communication: Fresh perspectives for molecular resistance breeding. Curr. Opin. Biotech. 14:177-193.

24. Holley, R. N., and Goodman, M. M. 1989. New sources of resistance to southern corn leaf blight from tropical hybrid maize derivatives. Plant Dis. 73:562-564.

25. Jenns, A. E., Leonard, K. J., and Moll, R. H. 1982. Variation in the expression of specificity in two maize diseases. Euphytica 31:269-280.

26. Jiang, J. C., Edmeades, G. O., Armstead, I., Lafitte, H. R., Hayward, M. D., and Hoisington, D. 1999. Genetic analysis of adaptation differences between highland and lowland tropical maize using molecular markers. Theor. Appl. Genet. 99:1106-1119.

27. Lim, S. M., and Hooker, A. L. 1976. Estimates of combining ability for resistance to Helminthosporium maydis race $\mathrm{O}$ in a maize population. Maydica 21:121-128.

28. McMullen, M. D., and Simcox, K. D. 1995. Genomic organization of disease and insect resistance genes in maize. Mol. Plant-Microbe Interact. 8:811-815.

29. Niks, R. E., Fernandez, E., Van-Haperen, B., Bekele Aleye, B., and Martinez, F. 2000. Specificity of QTLs for partial and non-host resistance of barley to leaf rust fungi. Acta Phytopathol. Hun. 35:13-21.

30. Parlevliet, J. E., and Van Ommeren, A. 1985. Race-specific effects in major genic and polygenic resistance of barley Hordeum vulgare to barley leaf rust in the field: identification and distinction. Euphytica 34:689-696.

31. Paterson, A. H., Damon, S., Hewitt, J. D., Zamir, D., Rabinowitch, H. D., Lincoln, S. E., Lander, E. S., and Tanksley, S. D. 1991. Mendelian factors underlying quantitative traits in tomato: Comparison across species, generations, and environments. Genetics 127:181-198.

32. Rathjen, J. P., and Moffett, P. 2003. Early signal transduction events in specific plant disease resistance. Curr. Opin. Plant Biol. 6:300-306.

33. Sallaud, C., Lorieux, M., Roumen, E., Tharreau, D., Berruyer, R., Svestasrani, P., Garsmeur, O., Ghesquiere, A., and Notteghem, J. L. 2003. Identification of five new blast resistance genes in the highly blastresistant rice variety IR64 using a QTL mapping strategy. Theor. Appl. Genet. 106:794-803.

34. Scott, G. E., and Futrell, M. C. 1975. Reaction of diallel crosses of maize in T and N cytoplasms in Bipolaris maydis race T. Crop Sci. 15:779-782.

35. Senior, M. L., Chin, E. C. L., Lee, M., Smith, J. S. C., and Stuber, C. W. 1996. Simple sequence repeat markers developed from maize sequences found in the GENBANK database: Map construction. Crop Sci. 36:16761683.

36. Thompson, D. L., and Bergquist, R. R. 1984. Inheritance of mature plant resistance to Helminthosporium maydis race 0 in maize Zea mays. Crop Sci. 24:807-811.

37. Ullstrup, A. J. 1972. The impact of the southern corn leaf blight epidemics of 1970-71. Annu. Rev. Phytopathol. 10:37-50.

38. Wang, S., Basten, C. J., and Zeng, Z.-B. 2004. Windows QTL Cartographer 2.0. Department of Statistics, North Carolina State University, Raleigh.

39. Welz, H. G., Schechert, A. W., and Geiger, H. H. 1999. Dynamic gene action at QTLs for resistance to Setosphaeria turcica in maize. Theor. Appl. Genet. 98:1036-1045.

40. Zaitlin, D., Demars, S., and Ma, Y. 1993. Linkage of rhm, a recessive gene for resistance to southern corn leaf blight, to RFLP marker loci in maize (Zea mays) seedlings. Genome 36:555-564.

41. Zhu, H., Braun, E. J., Perry, J. L., and Bronson, C. R. 1998. Identification, characterization, and mapping of Ecml, a locus affecting extracellular matrix production and lesion size in Cochliobolus heterostrophus. Genome 41:111-119. 